\title{
Geo-neutrinos, Mantle Circulation and Silicate Earth
}

\section{Gianni Fiorentini*}

Dipartimento di Fisica, Università di Ferrara, and Istituto Nazionale di Fisica Nucleare, Sezione di Ferrara, I-44100 Ferrara, Italy

E-mail: if iorenti@fe.infn. itt'

\section{Marcello Lissia}

Istituto Nazionale di Fisica Nucleare, Sezione di Cagliari, and Dipartimento di Fisica, Università di Cagliari, I-09042 Monserrato (CA), Italy

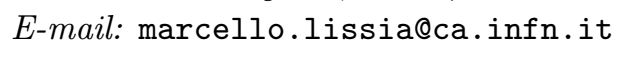

\section{Fabio Mantovani}

Dipartimento di Scienze della Terra, Università di Siena, I-53100 Siena, Italy Centro di GeoTecnologie CGT, I-52027 San Giovanni Valdarno, Italy

Istituto Nazionale di Fisica Nucleare, Sezione di Ferrara, I-44100 Ferrara, Italy

E-mail: 'mantovani@fe.infn. it'

\section{Riccardo Vannucci}

Dipartimento Scienze della Terra, Università di Pavia, I-27100 Pavia, Italy

E-mail: ivannucci@erysstal.unipv. ití

ABstRACT: In preparation to the experimental results which will be available in the future, we consider geo-neutrino production in greater detail than in [F. Mantovani et al., arXiv:hep-ph/0309013], putting the basis for a more refined model. We study geo-neutrino production for different models of matter circulation and composition in the mantle. By using global mass balance for the Bulk Silicate Earth, the predicted flux contribution from distant sources in the crust and in the mantle is fixed within $\pm 15 \%$ (full range). A detailed geological and geochemical investigation of the region near the detector has to be performed, for reducing the flux uncertainty from fluctuations of the local abundances to the level of the global geochemical error. A five-kton detector operating over four years at a site relatively far from nuclear power plants can measure the geo-neutrino signal with $5 \%$ accuracy $(1 \sigma)$. It will provide a crucial test of the Bulk Silicate Earth and a direct estimate of the radiogenic contribution to terrestrial heat.

Keywords: 'Neutrino Physics, Neutrino Detectors and Telescopes, Solar and" Āttmospheric Neutrinosi

\footnotetext{
${ }^{*}$ Speaker.
} 


\section{Introduction}

KamLAND, the Low Energy Anti-Neutrino Detector operating at the Kamioka mine in Japan, has demonstrated the possibility of detecting geo-neutrinos, the antineutrinos from decay chains of radiogenic nuclides inside Earth. A new window on Earth's interior

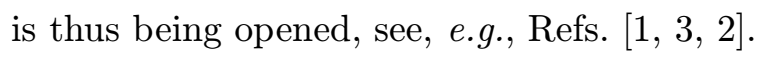

Recently, a reference model of geo-neutrino fluxes has been presented in Ref. [落] The model, hereafter indicated as REF, is based on a detailed description of Earth's crust and mantle, and takes into account available information on the abundances of Uranium, Thorium and Potassium - the most important heat and neutrino sources — inside Earth's layers.

This model has to be intended as a starting point, providing first estimates of expected events at several locations on the globe, see Fig. ${ }_{1}^{1}{ }^{1}$. In preparation to the experimental results which will be available in the future, from KamLAND as well as from other detectors which are in preparation, it is useful to consider geo-neutrino production in greater detail, putting the basis for a more refined model. In this respect, let us discuss critically the key ingredients of REF.

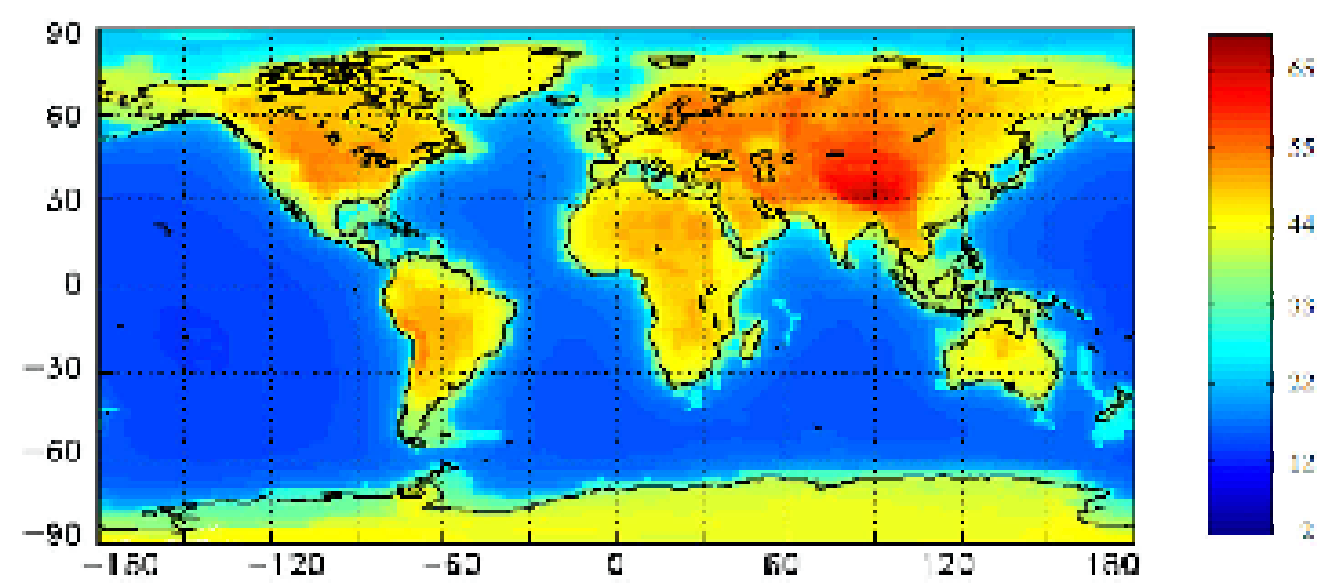

Figure 1: Predicted geo-neutrino events form $\mathrm{U}$ and Th decay chains from $\bar{\nu}_{e}+p \rightarrow e^{+}+n$ reactions, normalized to $10^{32}$ proton yr.

Concerning the crust, the $2^{\circ} \times 2^{\circ}$ model of Ref. [iיi] was adopted for building REF; worldaverage abundances $a$ of radiogenic elements have been estimated separately for oceans, the continental crust (subdivided into upper, middle and lower sub-layers), sediments, and oceanic crust. Although this treatment looks rather detailed on the globe scale, the typical linear dimension of each tile is of order $200 \mathrm{~km}$, so that any information on a smaller scale is essentially lost. Since one expects that about one third of the signal is contributed from sources within $100 \mathrm{Km}$ from the detector, a better description of the surrounding region is needed for a refined estimate.

\footnotetext{
${ }^{1}$ An interactive version of the geo-neutrino event map shown in Fig. $\bar{\eta}_{1}^{\prime}$ will be available at the site

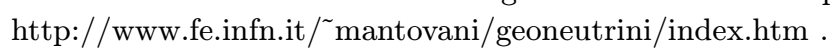


Sandwiched between Earth's crust and metallic core, the mantle is a $2900 \mathrm{~km}$ layer

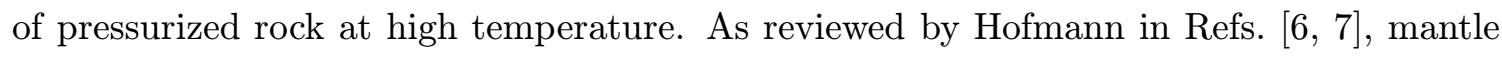
models can be divided into two broad classes, essentially corresponding to the presently contradictory geochemical and geophysical evidence of Earth's interior.

Geochemists have long insisted on a two-layer model, in which the mantle consists of a relatively primitive layer below a depth of about $670 \mathrm{~km}$ and an upper layer that is highly depleted of heat producing elements (panel "a" in Fig. viewed as separate sources of the Mid-Ocean-Ridge Basalts (MORB), which come from mantle regions that have been already depleted in incompatible elements by extraction of the continental crust, and of Ocean Island Basalts (OIB), which form by melting of deeper, less depleted or even enriched mantle sectors. Also, a more primitive deep layer is needed from global constraints, otherwise the amount of radiogenic elements inside Earth is much too small with respect to that estimated within the Bulk Silicate Earth (BSE) paradigm (see below).

On the other hand, over the past several years seismic tomography has provided increasingly detailed images of apparently cold slab descending into the deep mantle, below the $670-\mathrm{km}$ boundary. If cold slabs descend into the deep mantle, there must be a corresponding upward flow of deep-mantle material to shallow levels (panel "b" in Fig. '2i'). If this circulation reaches the bottom of the mantle (whole mantle convection), it would destroy any compositional layering below the crust in a few hundred million years (at a typical speed of $3 \mathrm{~cm} \mathrm{yr}^{-1}$ it takes about $10^{8} \mathrm{yr}$ to move down to $2900 \mathrm{~km}$ ).

In REF, the Preliminary Reference Earth Model [9í] was used for the mantle density profile, dividing Earth's interior into several spherically symmetrical shells corresponding to seismic discontinuities. Concerning its composition, a two-layer stratified model was used: for present day upper mantle, considered as the source of MORB, mass abundances of 6.5 and $17.3 \mathrm{ppb}$ for Uranium and Thorium, respectively, and $78 \mathrm{ppm}$ for Potassium were assumed down to a depth $h_{0}=670 \mathrm{~km}$. These abundances were obtained by averaging the

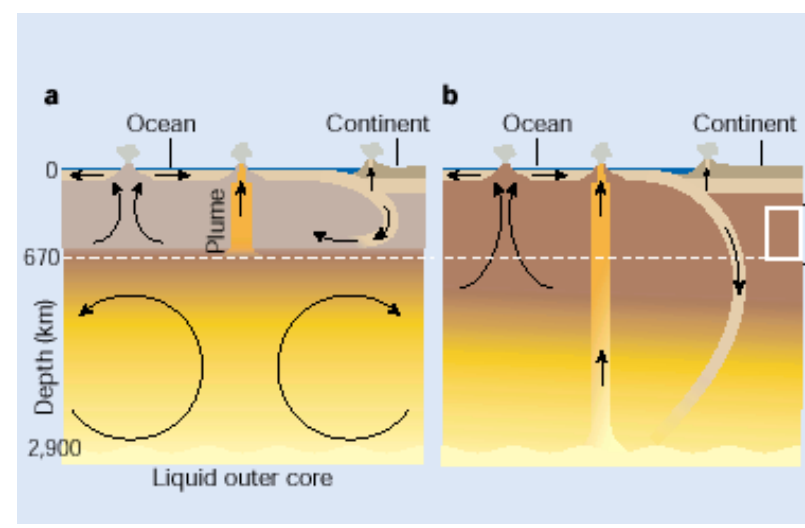

Figure 2: Models of mantle circulation, adapted from Ref. 187: (a) is the traditional two-layer model with demarcation at $670 \mathrm{~km}$ and nearly complete isolation between upper and lower layers; (b) is a fully mixed model.

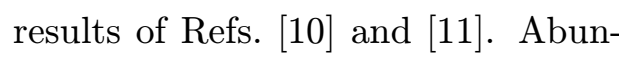
dances below were inferred by requiring that the BSE constraint is globally satisfied, obtaining 13.2 and $52 \mathrm{ppb}$ for $\mathrm{U}$ and Th, $160 \mathrm{ppm}$ for K. In view of the present debate about mantle circulation and composition, it is desirable to have a more general treatment, which encompasses both geochemical and geophysical supported models. 
The Bulk Silicate Earth framework provides a compositional description of the primitive mantle, the outer part of Earth after core separation and before crust differentiation. In this frame, the amount of heat/neutrino generating material inside Earth is determined through the following steps:

(a) From the compositional study of selected samples emerging from the mantle, after correcting for the effects of partial melting, one establishes the absolute primitive abundances in major elements with refractory and lithophile character, i.e., elements with high condensation temperature (so that they do not escape in the processes leading to Earth formation) and which do not enter the metallic core. In this way primitive absolute abundances of elements such as $\mathrm{Al}, \mathrm{Ca}$ and $\mathrm{Ti}$ are determined, a factor about 2.75 times $\mathrm{CI}$ chondritic abundances.

(b) It is believed, and supported by studies of mantle samples, that refractory lithophile elements inside Earth are in the same proportion as in chondritic meteorites. In this way, primitive abundances of Th and $U$ can be derived by rescaling the chondritic values ${ }^{2}$.

(c) Potassium, being a moderately volatile elements, could have escaped in the planetesimal formation phase. Its absolute abundance is best derived from the practically constant mass ratio with respect to Uranium observed in crustal and mantle derived rocks.

The BSE estimates for the total amounts of Uranium, Thorium and Potassium from

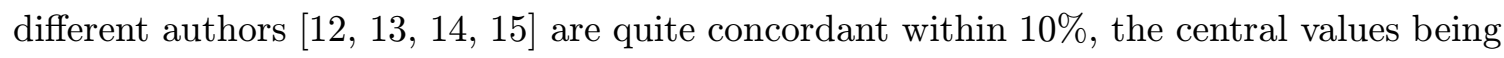
$m_{\mathrm{BSE}}=0.8 \times 10^{17} \mathrm{~kg}$ for Uranium, $3.1 \times 10^{17} \mathrm{~kg}$ for Thorium, and $0.9 \times 10^{21} \mathrm{~kg}$ for Potassium. These values can be taken - within their uncertainties - as representative of the composition of the present crust plus mantle system ${ }^{3}$.

Different models can provide different distributions between crust and mantle, however for each element the sum of the masses is fixed by the BSE constraint. This clearly provides constraints on the geo-neutrino flux ${ }^{4}$ which are grounded on sound geochemical arguments or, alternatively, geo-neutrino detection can provide a test of an important geochemical paradigm.

We remark that BSE predictions for the radiogenic contributions to Earth's flow are $H(\mathrm{U})=7.6 \mathrm{TW}, H(\mathrm{Th})=8.5 \mathrm{TW}$ and $H(\mathrm{~K})=1.8 \mathrm{TW}$, their sum being about one half of the observed heat flow from Earth, $H_{E} \approx 40 \mathrm{TW}$. Geo-neutrino detection can thus provide a direct insight on the main source of Earth's energetics, see [3i]|.

The reference model was built so as to satisfy the BSE constraint. However, when discussing its uncertainties a wide range of models was considered, from a minimal model providing a radiogenic contribution of just $9 \mathrm{TW}$ up to a fully radiogenic model. These extreme models, although not excluded from direct observational data, are well outside the BSE geochemical constraint. If the BSE global constraint is imposed and only the

\footnotetext{
${ }^{2}$ Note that Uranium absolute abundances in chondritic meteorites are variable within a factor two, however the ratio to Aluminum is stable within $10 \%$.

${ }^{3}$ Geochemists generally agree on the absence of these lithophile elements in Earth's core, see however Refs. [i 'is', '16i] for a different point of view.

${ }^{4}$ We are always speaking of the flux integrated over any direction, see [3] geo-neutrino flux.
} 
crust contribution is left free within the observational uncertainties, then the geo-neutrino prediction becomes much more precise.

Briefly, in this paper we shall address three questions.

(i) How sensitive are the predicted geo-neutrino fluxes to uncertainties about the mechanism of mantle circulation?

(ii) Is it possible to test the Bulk Silicate Earth model with neutrinos?

(iii) Which accuracy is needed concerning the local/regional predictions and the geoneutrino detection?

We shall restrict the discussion to geo-neutrinos from Uranium progeny, which are more easily detectable due to the higher energy. Extension to the other chains is immediate.

\section{Geochemistry, geophysics and geo-neutrinos}

As mentioned in the introduction, the composition and circulation inside Earth's mantle is the subject of a strong and so far unresolved debate between geochemists and geophysicists. Geochemistry supports the existence of two compositionally distinct reservoirs in the mantle, the borders between them being usually placed at depth near $h_{0}=670 \mathrm{~km}$, whereas geophysics presents evidence of matter circulation extending well beyond this depth. If this circulation involves the whole mantle, it would have destroyed any pre-existing layering, in conflict with geochemical evidence. In this section we look at the implications of this controversy on the predicted geo-neutrino fluxes.

One can build a wide class of models, including the extreme geochemical and geophysical models, in terms of just one free parameter, the depth $h$ marking the borders between the two hypothetical reservoirs:

(i) We assume that Uranium abundance in the uppermost part of the mantle is nearly chondritic ${ }^{5}\left(a_{u}=6.5 \mathrm{ppb}\right)$ down to an unspecified depth $h$.

(ii) Below $h$ we determine abundances $\left(a_{l}\right)$ by requiring mass balance for the whole Earth.

This means that Uranium mass below the critical depth, $m_{>h}$, is obtained by subtracting from the total BSE estimated mass $\left(m_{\mathrm{BSE}}\right)$ the quantity observationally determined in the crust $\left(m_{c}\right)$ and that contained in the mantle above $h\left(m_{<h}\right)$ :

$$
m_{>h}=m_{\mathrm{BSE}}-m_{c}-m_{<h}
$$

The abundance in the lower part is then calculated as the ratio of $m_{>h}$ to Earth's mass below $h\left(M_{>h}\right)$ :

$$
a_{l}=m_{>h} / M_{>h}
$$

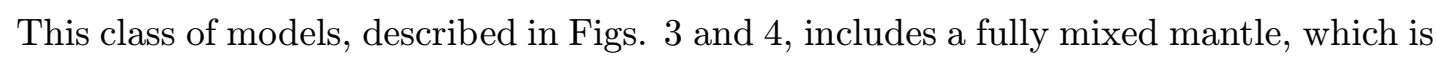
obtained for $h=25 \mathrm{~km}$ (i.e., just below the crust) so that the strongly impoverished mantle

\footnotetext{
${ }^{5}$ We are aware that current estimates of $U$ in depleted upper mantle after crust extraction are in the

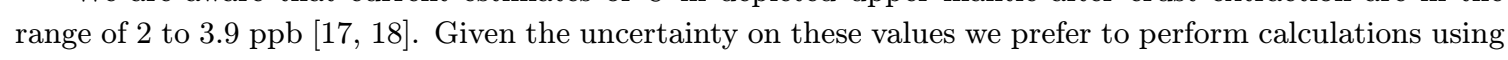
the well-constrained CI value. As shown below, the assumption of lower U abundance for the uppermost depleted mantle has limited effects on geo-neutrino flux predictions.
} 
has a negligible thickness. The traditional geochemical model corresponds to $h=h_{0}$. As $h$ increases, the depleted region extends deeper inside the Earth and - due to mass balance the innermost part of the mantle becomes richer and closer in composition to the primitive mantle.

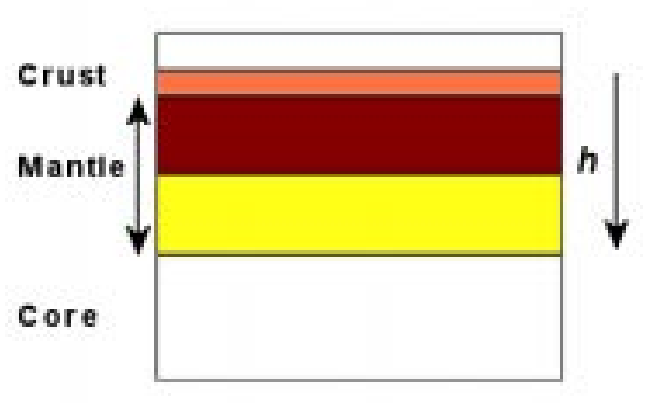

Figure 3: Generic two-reservoir mantle model. Uranium abundance in the upper part is fixed at $a_{u}=6.5 \mathrm{ppb}$, the critical depth $h$ is a free parameter, abundance in the lower part $a_{l}$ is determined for a fixed total Uranium mass in the mantle $m_{m}=$ $0.45 \times 10^{17} \mathrm{~kg}$.

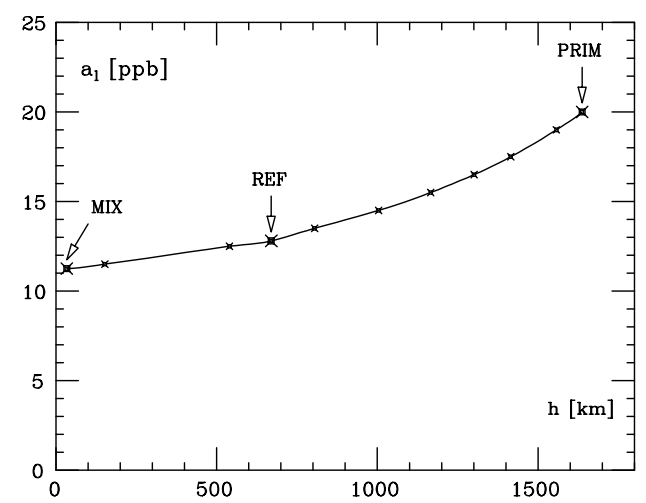

Figure 4: Uranium abundance in the lower part of the mantle $a_{l}$ as function of the critical depth $h$ from Earth's surface.

Let us discuss in detail a few cases, remembering that the BSE estimate for Uranium in the whole Earth is $m_{\mathrm{BSE}}=0.8 \times 10^{17} \mathrm{~kg}$ and that the best estimate for the amount in the crust is $\left.m_{c}=0.35 \times 10^{17} \mathrm{~kg}\left[i_{i}^{i}\right]\right]$ so that Uranium in the mantle is $m_{m}=0.45 \times 10^{17} \mathrm{~kg}$.

(a) In the fully mixed model, this quantity has to be distributed over the mantle mass $M_{m}=4.0 \times 10^{24} \mathrm{~kg}$, which yields a uniform mantle abundance $a=11.25 \mathrm{ppb}$. We shall refer to this model as MIX.

(b) If we keep the estimated abundance in the uppermost part $\left(a_{u}=6.5 \mathrm{ppb}\right)$ down to $h_{0}$ one has the REF model [4]-

(c) Among all possible models, the case $h=1630 \mathrm{~km}$ is particularly interesting. Below this depth the resulting Uranium abundance is $20 \mathrm{ppb}$, corresponding to the BSE estimate. The innermost part of the mantle is thus primitive in its trace element composition and the crust enrichment is obtained at expenses of the mantle content above $h$. We shall refer to this model as PRIM.

Concerning geo-neutrino fluxes from the mantle, all these models have the same amount of heat/neutrino sources and only the geometrical distribution is varied. The largest flux corresponds to the model with more sources close to the surface, i.e. to the MIX model. On the other hand, the minimal prediction is obtained when the sources are most concentrated at larger depth, which corresponds to the PRIM case. 
From Table the extreme cases is $8 \%$, model REF being in between. The abundance in the upper reservoir $a_{u}$ can also be treated as a free parameter. If we use an extremely low value $a_{u}=2 \mathrm{ppb}[\underline{1} \overline{1} \overline{\mathbf{1}}]$ ] down to about $1200 \mathrm{~km}$ and primitive abundance below, we obtain the minimal prediction $0.86 \times 10^{6} \mathrm{~cm}^{-2} \mathrm{~s}^{-1}$.

We conclude this section with the following remarks:

\begin{tabular}{lcc}
\hline Model & $\begin{array}{c}\text { Critical depth } h \\
{[\mathrm{~km}]}\end{array}$ & $\begin{array}{c}\text { Flux } \\
{\left[10^{6} \mathrm{~cm}^{-2} \mathrm{~s}^{-1}\right]}\end{array}$ \\
\hline \hline MIX & 25 & 1.00 \\
REF & 670 & 0.95 \\
PRIM & 1630 & 0.92 \\
\hline
\end{tabular}

Table 1: Mantle contribution to the produced Uranium geo-neutrino flux. The same Uranium mass in the mantle $m_{m}=0.45 \times 10^{17} \mathrm{~kg}$ and abundance in the upper layer $a_{u}=6.5 \mathrm{ppb}$ are assumed in each model.

- uncertainties on the geometrical distribution of trace elements in the mantle can change the REF prediction for the mantle by at most $\pm 8 \%$.

- A geo-neutrino detector at a site where the contribution from the mantle is dominant (i.e., far from the continental crust) can be sensitive to the mantle compositional geometry only if a percent accuracy is reached.

- Since at Kamioka mine or at Gran Sasso the mantle contribution to the total flux is about one quarter of the total [inj], uncertainties on the mantle geometry imply an estimated error of about $2 \%$ on the total flux predicted with REF.

\section{The Bulk Silicate Earth constraint}

So far we have been considering the effect of different geometrical distributions of trace elements in the mantle, for fixed amount of these elements within it. Actually the BSE model can be exploited to obtain tight constraints on the total flux produced together from the crust and the mantle. In fact, with BSE fixing the total amount of trace elements inside Earth, geometrical arguments and observational constraints on the crust composition can be used in order to find extreme values of the produced fluxes. As an extension of the previous section, the maximal (minimal) flux is obtained by placing the sources as close (far) as possible to Earth's surface, where the detector is located.

Concerning Uranium, the range of BSE concentrations reported in the literature is between 18 and $23 \mathrm{ppb}$, corresponding to a total Uranium mass between $m(\min )=0.72$ and $m(\max )=0.92$ in units of $10^{17} \mathrm{~kg}$.

In the same units, we estimate that Uranium mass in the crust is between $m_{c}(\min )=$ 0.30 and $m_{c}(\min )=0.41$, by taking the lowest (highest) concentration reported in the literature for each layer, see Table II of [i⿶凵]. The main source of uncertainty is from the

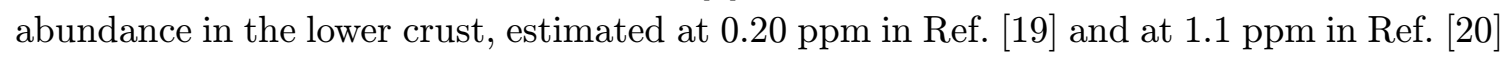
Estimates for the abundance in the upper crust are more concordant, ranging from 2.2

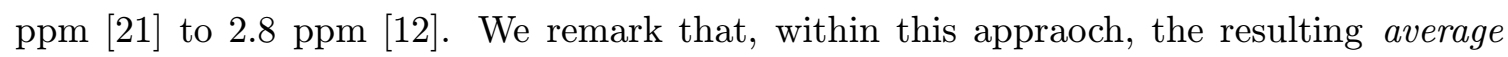
crustal $\mathrm{U}$ abundance $\left\langle a_{c c}\right\rangle$ is in the range 1.3-1.8 ppm, which encompasses all estimates 


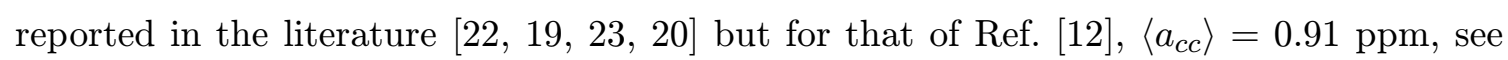
Table ${ }_{2}^{2}{ }_{i}^{6}$.

The highest flux is obtained by assuming the maximal mass in the crust and the maximal mass in the mantle, $m_{\text {tot }}(\max )-m_{c}(\max )=0.51$, with a uniform distribution inside the mantle, corresponding to $a=12.8 \mathrm{ppb}$.

On the other hand, the lowest flux corresponds to the minimal mass in the crust and the minimal mass in the mantle, $m_{\text {tot }}(\min )-m_{c}(\min )=0.42$, with a distribution in the mantle similar to that of PRIM, i.e. a strongly

\begin{tabular}{|c|c|}
\hline Reference & $\left\langle a_{c c}\right\rangle[\mathrm{ppm}]$ \\
\hline Taylor \& Mclennan $1985[1]$ & 0.91 \\
\hline Weaver \& Tarney 1984 [i2 $2 \overline{2}]$ & 1.3 \\
\hline Rudnick \& Fountain $1995[1]$ & 1.42 \\
\hline 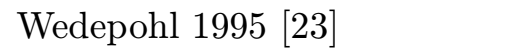 & 1.7 \\
\hline 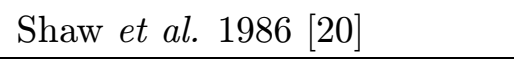 & 1.8 \\
\hline This work, minimal & 1.3 \\
\hline This work, reference & 1.54 \\
\hline This work, maximal & 1.8 \\
\hline
\end{tabular}

Table 2: Average Uranium abundance in the continental crust. depleted mantle with $a_{u}=2 \mathrm{ppb}$ down to about $1300 \mathrm{~km}$ and a primordial composition beneath.

The predicted fluxes are shown in Table 'i3i-1 for a few locations of particular interest: the Kamioka mine $\left(33^{\circ} \mathrm{N} 85^{\circ} \mathrm{E}\right)$ where KamLAND is operational, the Gran Sasso laboratory $\left(42^{\circ} \mathrm{N} 14^{\circ} \mathrm{E}\right)$ where BOREXINO [2, $\left.2 \overline{4} 4\right]$ is in preparation, the top of Himalaya $\left(36^{\circ} \mathrm{N}\right.$ $\left.137^{\circ} \mathrm{E}\right)$, which receives the maximal contribution from the crust, and Hawaii $\left(20^{\circ} \mathrm{N} 156^{\circ} \mathrm{E}\right)$, a location where the mantle contribution is dominant.

\begin{tabular}{lcccccc}
\hline & \multicolumn{1}{c}{ Himalaya } & $\begin{array}{c}\text { Gran Sasso } \\
\Phi\end{array}$ & Kamioka & Hawaii \\
& $m$ & $H$ & \multicolumn{5}{c}{2.84} & 2.35 & 0.33 \\
\hline \hline Crust MIN & 0.30 & 2.85 & 4.92 & 3.27 & 2.73 & 0.37 \\
Crust REF & 0.35 & 3.35 & 5.71 & 3.74 & 3.13 & 0.42 \\
Crust MAX & 0.41 & 3.86 & 6.55 & 0.80 & & \\
Mantle MIN & 0.42 & 3.99 & & 0.95 & & \\
Mantle REF & 0.45 & 4.29 & & 1.14 & & \\
Mantle MAX & 0.51 & 4.84 & & 3.64 & 3.15 & 1.13 \\
\hline Total MIN & 0.72 & 6.84 & 5.72 & 4.22 & 3.68 & 1.32 \\
Total REF & 0.80 & 7.64 & 6.66 & 4.88 & 4.27 & 1.54 \\
Total MAX & 0.92 & 8.70 & 7.69 & & & \\
\hline
\end{tabular}

Table 3: Produced Uranium geo-neutrino fluxes within BSE. Minimal and maximal fluxes are shown, together with the Reference values of Ref. [4] rate $H$ within each layer are also presented. Units for mass, heat flow and flux are $10^{17} \mathrm{~kg}$, TW and $10^{6} \mathrm{~cm}^{-2} \mathrm{~s}^{-1}$, respectively.

At any site the difference between the maximal and the minimal flux predictions are of about $30 \%$, the extreme values being within $\pm 15 \%$ from the reference model prediction.

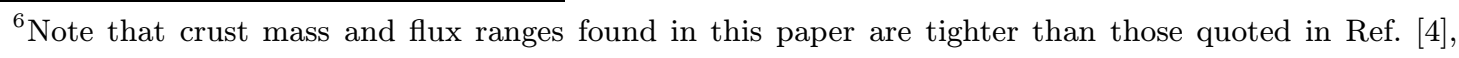
which used the value $\left\langle a_{c c}\right\rangle=0.91 \mathrm{ppm}$ from Ref. [1]
} 
All this shows the power of the BSE constraint. If the total amount of Uranium inside Earth is fixed at $m_{\mathrm{BSE}}=(0.8 \pm 0.1) \times 10^{17} \mathrm{~kg}$, then the produced geo-neutrino flux at, e.g., Kamioka is

$$
\Phi=(3.7 \pm 0.6) \times 10^{6} \mathrm{~cm}^{-2} \mathrm{~s}^{-1} \quad \text { (full range) }
$$

after taking into account the full range of global observational uncertainties on Uranium abundances in the crust and uncertainties concerning circulation in the mantle.

For a comparison, we remind that in Ref. [i] vary in the range $(0.4-1.7) \times 10^{17} \mathrm{~kg}$, the whole range uncertainty on the produced flux at Kamioka was $\pm 1.9 \times 10^{6} \mathrm{~cm}^{-2} \mathrm{~s}^{-1}$.

We remark that the error quoted in Eq. (3. values. If, following a commonly used rule of thumb, we consider such a range as a $\pm 3 \sigma$ $(99.5 \%)$ confidence level, we deduce a conventional $1 \sigma$ estimate:

$$
\Phi=(3.7 \pm 0.2) \times 10^{6} \mathrm{~cm}^{-2} \mathrm{~s}^{-1} \quad(1 \sigma) .
$$

\section{The effects of geochemical fluctuations and neutrino oscillations}

The main result of the previous section is that — neglecting regional fluctuations - global mass balance provides a precise determination of the produced geo-neutrino fluxes. We shall compare this precision with uncertainties resulting from fluctuations of the regional geochemical composition, from the available information on neutrino mixing parameters and from the detector finite size.

(a) Actually the Uranium concentration in the region where the detector is located can be different from the world average and local fluctuations of this highly mobile element can be envisaged. These variations, although negligible for mass balance, can affect significantly the flux. In other words, geochemical arguments fix the contribution of distant sources, and a more detailed geological and geochemical investigation of the region around the detector is needed, the error quoted in Eq. $\left(\overline{3} \cdot \overline{2}_{1}\right)$ providing a benchmark for the accuracy of the local evaluation.

As an example, it has been estimated that about one third (one half) of the geoneutrino signal is generated within a distance of 100 (500) km from Kamioka, essentially in the Japanese continental shelf. In REF the world averaged upper crust Uranium concentration $a_{u c}=2.5 \mathrm{ppm}$ was adopted for Japan. In a recent study of the chemical composition of Japan upper crust [2] $2 \overline{5}]$ more than hundred samples, corresponding to 37 geologic groups, have been analyzed. The composition is weighted with the frequency in the geological map and the resulting average abundance is $a_{\mathrm{Jap}}=2.32 \mathrm{ppm}$, which implies a $7.2 \%$ reduction of the flux from Japanese upper crust with respect to the estimate in REF. Larger variations occur when rocks are divided according to age or type, see Table 佟, and even larger differences are found within each group. All this calls for a detailed geochemical and geophysical study, with the goal of reducing the effect of regional fluctuations to the level of the uncertainty from global geochemical constraints.

(b) Let us remark that the signal is originated from neutrinos which maintain the electron flavour in their trip from source to detector, the effective flux being $\Phi_{\text {eff }}=\Phi P_{e e}$, 


\begin{tabular}{lcc}
\hline Group & Area (\%) & $a$ (ppm) \\
\hline \hline Pre-Neogene & 41.7 & 2.20 \\
Pre-Cretaceous & 10.5 & 2.11 \\
Neog-Quat. Igneous rocks & 24.1 & 2.12 \\
Paleog-Cret. Igneous rocks & 14.1 & 3.10 \\
\hline sedimentary & 39.9 & 2.49 \\
metamorphic & 21.3 & 1.72 \\
igneous & 38.4 & 2.48 \\
\hline Global area weighted average & 99.6 & 2.32 \\
\hline
\end{tabular}

Table 4: Uranium abundance in the upper continental crust of Japan. Groups correspond to rock's age or type and quoted abundances for each group are area weighted values, from Ref. [2 25$]$.

where $\Phi$ is the produced flux and $P_{e e}=1-1 / 2 \sin ^{2}(2 \theta)$ is the (distance averaged) survival probability. From the analysis of all available solar and reactor neutrino experiments, one gets $\tan ^{2} \theta=0.41 \pm 0.04$ at $1 \sigma[\overline{2} \overline{6} \overline{0}]$, which implies:

$$
P_{e e}=0.59 \pm 0.02 \quad(1 \sigma) \quad .
$$

The survival probability is presently know with a $3 \%$ accuracy, so that uncertainties on the neutrino fate do not mask its sources.

(c) If Uranium geo-neutrinos are detected by means of inverse beta reaction on free hydrogen nuclei $\left(\bar{\nu}_{e}+p \rightarrow e^{+}+n\right)$ the event number is [i2 2$]$

$$
N=13.2 \epsilon \frac{\Phi_{\text {eff }}}{10^{6} \mathrm{~cm}^{-2} \mathrm{~s}^{-1}} \frac{N_{p}}{10^{32}} \frac{t}{\mathrm{yr}},
$$

where $\epsilon$ is the detection efficiency, $N_{p}$ is the number of free protons in the target and $t$ is the measurement time. For a produced flux $\Phi=4 \times 10^{6} \mathrm{~cm}^{-2} \mathrm{~s}^{-1}$ and $\epsilon=0.8$, one expects 25 events for an exposure of $10^{32}$ protons yr. Statistical fluctuations will be of order $\sqrt{N}$ if background can be neglected (this clearly does not hold for Kamioka, due to the many nearby nuclear power plants). In order to reach a $5 \%$ accuracy - comparable to that of the global geochemical estimate - one needs an exposure of $16 \times 10^{32}$ protons yr, which corresponds to a five-kton detector operating over four years.

\section{Concluding remarks}

We summarize here the main points of this paper:

(1) uncertainties on the geometrical distribution of trace elements in the mantle (for a fixed mass within it) can change the prediction of the reference model [4] for the geoneutrino flux from mantle by at most $\pm 8 \%$ (full range), the extreme values corresponding to a fully-mixed and to a two-layer model, with primordial abundance below about 1300 $\mathrm{km}$.

(2) By using global mass balance for the Bulk Silicate Earth, the predicted flux contribution originating from distant sources in the crust and in the mantle is fixed within $\pm 5 \%$ $(1 \sigma)$ with respect to the reference model. 
(3) A detailed geological and geochemical investigation of the region within few hundreds $\mathrm{km}$ from the detector has to be performed, for reducing the flux uncertainty from fluctuations of the local abundances to the level of the global geochemical error.

(4) A five-kton detector operating over four years at a site relatively far from nuclear power plants can measure the geo-neutrino signal with $5 \%$ accuracy.

(5) This will provide a crucial test of the Bulk Silicate Earth and a direct estimate of the radiogenic contribution to terrestrial heat.

\section{Acknowledgments}

We express our gratitude for useful discussions Dr. C. Bonadiman, L. Carmignani, M. Coltorti, S. Enomoto, K. Inoue, E. Lisi, T. Mitsui, B. Ricci, N. Sleep, A. Suzuki, and F. Villante. G. F. is grateful to the organizing committee of AHEP 2003 for a very enjoiable conference. This work was partially supported by MIUR (Ministero dell'Istruzione, dell'Università e della Ricerca) under MIUR-PRIN-2003 project "Theoretical Physics of the Nucleus and the Many-Body Systems" and MIUR-PRIN-2002 project "Astroparticle Physics".

\section{References}

[1] KamLAND Collaboration, K. Eguchi et al., Phys. Rev. Lett. 90 (2003) 021802 [arXiv:hep-ex/0212021].

[2] G. Fiorentini, T. Lasserre, M. Lissia, B. Ricci and S. Schonert, Phys. Lett. B 558 (2003) 15 [arXiv:hep-ph/0301042].

[3] G. Fiorentini, F. Mantovani and B. Ricci, Phys. Lett. B 557 (2003) 139 [arXiv:nucl-ex/0212008].

[4] F. Mantovani, L. Carmignani, G. Fiorentini and M. Lissia, Phys. Rev. D 69 (2004) 013001 [arXiv:hep-ph/0309013].

[5] C. Bassin, G. Laske. and G. Masters, EOS Trans. Am. Geophys. Union 81, F897 (2000) [http://mahi.ucsd.edu/Gabi/rem.html].

[6] A. W. Hofmann, Nature 385 (1997) 219.

[7] A. W. Hofmann, Nature 425 (2003) 24.

[8] V. Rama Murthy, Win Van Westrenen, and Yingwei Fei, Nature (London) 423 (2003) 163.

[9] A. M. Dziewonski and D. L. Anderson, Phys. Earth Planet. Interact. 25, 297 (1981).

[10] K. P. Jochum et al., Nature (London) 322, 221 (1986).

[11] R. E. Zartman and S. Haines, Geochim. Cosmochim. Acta 52, 1327 (1988).

[12] S. R. Taylor and S. M. McLennan, The Continental Crust: its Composition and Evolution (Blackwell Scientic, Oxford, 1985).

[13] A. W. Hofmann, Earth Planet Sci. Lett. 90 (1088) 297.

[14] W. F. McDonough, S. S. Sun, A. E. Ringwood, E. Jagoutz, and A. W. Hofmann, Geochim. Cosmochim. Acta 56 (1992) 1001. 
[15] H. Wänke, G. Dreibus, and E. Jagoutz, "Mantle Chemistry and accretion history of the Earth," in Archean Geochemistry, edited by A. Kroener, G N. Hänson, and A. M. Goodwun (Springer-Verlag, New York, 1984), pp. 1-24.

[16] C. K. Gessmann and B. J. Wood, Earth and Planetary Science Letters, 200 (2002) 63.

[17] F. Albarède, Goechemistry. An Introduction. (Cambridge University Press, 2003)

[18] S.-s. Sun and W. F. McDonough, Chemical and isotopic systematics of oceanic basalts: implications for mantle composition and processes, in Magmatism in the oceanic basins, edited by A. .D. Saunders and M. J. Norry, Geological Society, Special Publication 42 (1989) pp. 313-345.

[19] R. L. Rudnick and D. M. Fountain, Rev. Geophys. 33 (1995) 267.

[20] D. M. Shaw, J. J. Cramer, M. D. Higgins, and M. G. Truscott, in The Nature of the Lower Continental Crust, edited by J. Dawson et al. (Geological Society of London, London, 1986), p. 275.

[21] K. C. Condie, Chem. Geol. 104 (1993) 1.

[22] B. L. Weaver and J. Tarney, Nature (London) 310 (1984) 575.

[23] K. H. Wedepohl, Geochim. Cosmochim. Acta 59, 1217 (1995).

[24] BOREXINO Collaboration, G. Alimonti et al., Astropart. Phys. 16 (2002) 205.

[25] S. Togashi et al., Geochem. Geophys. Geosyst. (Electronic Journal of the Earth Sciences) 1 (2000) 2000GC00083.

[26] J. N. Bahcall and C. Pena-Garay, JHEP 0311 (2003) 004 [arXiv:hep-ph/0305159]. 